\title{
Daily blood pressure profile and blood-brain barrier permeability in patients with cerebral small vessel disease
}

\section{A. Dobrynina}

Research Center of Neurology

K. V. Shamtieva ( $\square$ kamila.shamt@gmail.com )

Research Center of Neurology

E. I. Kremneva

Research Center of Neurology

M. R. Zabitova

Research Center of Neurology

B. M. Akhmetzyanov

Research Center of Neurology

E. V. Gnedovskaya

Research Center of Neurology

\section{V. Krotenkova}

Research Center of Neurology

\section{Research Article}

Keywords:

Posted Date: February 17th, 2022

DOI: https://doi.org/10.21203/rs.3.rs-1332879/v1

License: (9) (i) This work is licensed under a Creative Commons Attribution 4.0 International License.

Read Full License

Version of Record: A version of this preprint was published at Scientific Reports on May 11th, 2022. See the published version at https://doi.org/10.1038/s41598-022-11172-1. 


\section{Abstract}

\section{Background}

Cerebral small vessel disease (CSVD) plays an important role in cognitive impairment (Cl), stroke, disability and death. Arterial hypertension (AH) is the main risk factor for CSVD. The use of antihypertensive therapy (AHT) has not resulted in the expected decrease in CSVD complications, which may be related to the underestimation of significance of daily blood pressure profile for blood-brain barrier (BBB) permeability.

\section{Methods}

53 patients with CSVD of varying severity (mean age 60.086.8 years, 69.8\% women, subjects with longstanding $\mathrm{AH}$ vs. normotensive subjects $-84.8 \%$ vs. $15.2 \%$ ) and 17 healthy volunteers underwent ambulatory blood pressure monitoring (ABPM) and MRI, including T1-weighted dynamic contrastenhanced magnetic resonance imaging (DCE-MRI) for assessing BBB permeability.

Results

Most of ABPM parameters in CSVD patients did not differ from controls, but were associated with the severity of white matter hyperintensity $(\mathrm{WMH})$ and the total CSVD score. BBB permeability in normalappearing white matter (NAWM) and grey matter (GM) was significantly higher in CSVD patients, and the severity of BBB permeability remained similar in patients with different stages of WMH. Among BBB permeability parameters, the area under the curve, corresponding to an increase in the contrast transit time in NAWM, had the greatest number of correlations with deviations of ABPM parameters.

Conclusion

BBB permeability in CSVD is a universal mechanism of NAWM and GM damage associated with a slight increase in ABPM parameters. It is obvious that the treatment of $A H$ in patients with not severe WMH should be more aggressive and carried out under the control of ABPM.

\section{Introduction}

Cerebral small vessel disease (CSVD), associated with age and vascular risk factors, is the main cause of vascular cognitive impairment $(\mathrm{Cl})$, mixed neurodegenerative and vascular dementia, as well as a significant cause of stroke, disability and mortality $[1 ; 2 ; 3]$.

Arterial hypertension $(\mathrm{AH})$ is the main risk factor for age-related CSVD $[4 ; 5 ; 6]$. The link between $A H$ and CSVD has been confirmed morphologically $[7 ; 8]$ and experimentally $[9 ; 10]$ through the correlation between $\mathrm{AH}$ severity and the intensity of the diagnostic MRI signs of CSVD, such as white matter hyperintensities (WMH) and lacunes [4; 11], and the clinical symptoms, such as $\mathrm{Cl}$ and stroke $[12 ; 13]$. 
The leading mechanism of brain damage in patients with chronic $\mathrm{AH}$ is hypoperfusion secondary to arteriolosclerosis, which is characterized by the loss of smooth muscle cells, accumulation of fibrotic hyaline deposits, thickening of the blood vessel walls, and luminal narrowing [14; 15].

Antihypertensive therapy (AHT), which acts predominantly by preventing small artery remodelling and increasing blood flow, has led to changes in the progression of $\mathrm{AH}$ and its cerebral complications. Recent studies have noted the importance of blood pressure (BP) variability in the development of CSVD $[16 ; 17]$ and a decrease in the incidence of stroke owing to the AHT control of BP [18; 19]. At the same time, AHT has not ensured the expected decrease in the prevalence of $\mathrm{Cl}[20 ; 21]$. One of the explanations for the insufficient effectiveness of $\mathrm{AHT}$ in preventing $\mathrm{Cl}$ could be a lack of or a faint effect on blood-brain barrier (BBB) [22]. BBB damage is supposed to be a core mechanism in the initiation and progression of CSVD and $\mathrm{Cl}$ associated with the last one $[23 ; 24]$ as well as $\mathrm{Cl}$ secondary to mixed neurodegenerative and vascular dementia [25]. In these cases, AH is both a factor in BBB damage [26] and a consequence of the damage to the cerebral autonomic centres caused by high BBB permeability [27].

For a long time, BBB damage with high permeability has been considered mainly as a failure of cerebral autoregulation due to high BP in acute and chronic AH [28; 29]. Further experiments on the spontaneously hypertensive rats and stroke-prone spontaneously hypertensive rats have proven that the mechanism of BBB damage in CSVD is universal and can be observed in milder AH stages [30], similar to long-standing $\mathrm{AH}$. The importance of this mechanism in the development of CSVD was confirmed during in immunohistochemistry of patient brain samples where it was shown that the endothelial activation and passage of fluid through the BBB had led to the brain oedema and neuroinflammation [15; 31].

In vivo study of the role of this mechanism in CSVD development has become possible with T1-weighted dynamic contrast-enhanced magnetic resonance imaging (DCE-MRI), enabling a quantitative assessment of BBB permeability [32; 33]. Increased BBB permeability was found in normal-appearing white matter (NAWM) as compared to controls [34; 35]; in WMH and adjacent NAWM, correlating with the severity of $\mathrm{WMH}, \mathrm{AH}$, age and leading to the delayed reduction in cognitive capacity [32].

The obtained data on the importance of increased BBB permeability in the development of CSVD requires clarification in terms of the effect of AH on BBB permeability in patients with CSVD.

The study aim is to evaluate the relation between daily blood pressure profile and BBB permeability in patients with CSVD.

\section{Materials And Methods}

The study included patients aged 46-70 years with cognitive and other cerebral complaints, brain changes on MRI corresponded to CSVD (WMH, lacunes, enlarged perivascular spaces, microbleeds and cerebral atrophy) [36]. Patients with low WMH burden (Fazekas scale score 1) were included in the study if they had $\mathrm{AH}$ stage 2 or 3 and/or $\geq 1$ lacuna. 
Exclusion criteria: 1) Cl due to probable Alzheimer's disease according to the U.S. National Institute on Aging criteria [37; 38]; 2) patients with small subcortical infarcts/lacunes $<3$ months after an acute cerebrovascular event; 3 ) CSVD due to other independent causes (genetic, inflammatory, thrombophilic, systemic, toxic, history of severe migraines); 4) a different cause of stroke and concomitant brain pathology other than CSVD; 5$)>50 \%$ atherosclerotic stenosis of the extra- or intracranial arteries; 6 ) serious medical condition - cardiac (ejection fraction $<50 \%$ ), endocrine (diabetes mellitus (DM) type 1 or 2 with severe vascular complications, uncompensated thyroid disorder), renal (chronic kidney disease with glomerular filtration rate $<30 \mathrm{ml} / \mathrm{min}$ ), etc.; 7) contraindications for MRI.

The control group consisted of volunteers with no clinical or MRI evidence of vascular and degenerative brain pathology, no AH in the medical history and during Ambulatory Blood Pressure Monitoring (ABPM), and matched for age and gender. Controls with $\mathrm{AH}$ according to ABPM were excluded from the study, in accordance with the European Society of Hypertension recommendations: daytime BP was $\geq 135 / 85$ $\mathrm{mmHg}$ and/or night-time BP was $\geq 120 / 70 \mathrm{mmHg}$, or if BP increased by more than $24 \%$ over time during exertion [39].

In total 53 patients ( 37 women, average age $60.1 \pm 6.8$ years) and 17 healthy volunteers ( 12 women, average age $56.7 \pm 6.7$ years) were enrolled in the study. The study was approved by the Local Ethics Committee of the Research Centre of Neurology № №2-4/16 dated 17.02.2016 and performed in accordance with the principles of the Declaration of Helsinki. All subjects signed an informed consent form for participation in the study.

Traditional vascular risk factors, such as $\mathrm{AH}$ [40], hypercholesterolemia, obesity, DM and smoking were assessed in the patients and controls.

All participants underwent ABPM with an automated device (LLC DMS Advanced Technologies, Moscow) based on oscillometric method. Patients underwent ABPM during hospitalization with BP measurement every 30 min during the day (8:00 am to $10: 00 \mathrm{pm})$ and every $60 \mathrm{~min}$ during the night (10:00 pm to 8:00 am). The ABPM device inflatable cuff was placed on the non-dominant upper limb. In all cases, at least $70 \%$ of the measurements were suitable for analysis. We calculated mean 24 -hour systolic BP (SBP) and diastolic BP (DBP); mean, standard deviation (SD) and maximal values of awake and asleep SBP and DBP; and BP load parameters as the percentage of readings in a given period (24-h, day, or night), which exceed the normal levels for awake and asleep SBP and DBP [39].

The grade of AH was determined from the medical history and was adjusted according to ABPM results. During hospitalization patients continued their AHT.

Imaging was carried out in a Siemens MAGNETOM Verio 3T scanner (Siemens Medical Systems, Erlangen, Germany) with a standard 12-channel matrix head coil. To evaluate STRIVE criteria [36], patients and the control group underwent axial spin echo T2-weighed imaging (TR $4000 \mathrm{~ms}$; TE $118 \mathrm{~ms}$; slice thickness 5.0 mm; duration: 2 min $02 \mathrm{~s}$ ); sagittal 3D T2 FLAIR (TR $6000 \mathrm{~ms}$; TE $395 \mathrm{~ms} ; 1.0 \mathrm{~mm}^{3}$ cubic voxel; duration: 7 min 12 s); sagittal 3D T1-mpr (TR 1900 ms; TE 2,5 ms; 1.0 mm³ cubic voxel; 
duration: 4 min $16 \mathrm{~s}$ ); diffusion MRI (DWI) using axial spin-echo echo-planar imaging sequence with two b-values - 0, $1000 \mathrm{~s} / \mathrm{mm}^{2}$ (TR - $4000 \mathrm{~ms}$, TE - $100 \mathrm{~ms}$, slice thickness - $4 \mathrm{~mm}$, duration: $-1 \mathrm{~min} 20 \mathrm{~s}$ ); axial susceptibility weighted imaging sequence (SWI) with magnitude and phase images reconstruction (TR 28 ms; TE 20 ms; slice thickness 1.2 mm; duration: 8 min $12 \mathrm{~s}$ ).

Two neuroradiologists evaluated MR images in a standardized manner, blinded to clinical information. No STRIVE criteria were found in volunteers from control group. There were no acute or recent small lacunar infarcts based on DWI analysis in patients with CSVD. MRI presence of lacunes, white matter hyperintensities, microbleeds, and perivascular spaces were summed in a score of $0-4$ representing all SVD features combined [41; 42].

The Fazekas Scale [43] was used to quantify T2 FLAIR white matter hyperintensities (WMH) (score 0-3) as well as semi-automatic WMHs segmentation using LST toolbox (http://www.appliedstatistics.de/lst.htm) for SPM12 (http://www.fil.ion.ucl.ac.uk/spm) with further manual correction using ITK-SNAP viewer (http://itksnap.org). The obtained data were saved as a binary mask, which was taken into consideration when the NAWM mask was subsequently created to calculate BBB permeability.

DCE-MRI was performed for BBB leak assessment: after two T1 volumetric interpolated breath-hold examination (T1-VIBE) acquisitions (flip angles 2 and 15) for pre-contrast T1 maps, we injected gadodiamide (Omniscan; GE Healthcare) $0.2 \mathrm{~mL} / \mathrm{kg}$ (i.e., $0.1 \mathrm{mmol} / \mathrm{kg}$ body weight) at a rate 3 $\mathrm{mL} /$ second intravenously via injection pump and then repeated the 3D T1-weighted sequence sequentially 100 times for $15 \mathrm{~min} 33 \mathrm{sec}$. The scanning parameters were: TR $-8.6 \mathrm{msec}$, TE $-4 \mathrm{msec}$, field of view - $250 \mathrm{~mm}$, matrix - 256x230 pixels, flip angle -15 degrees, slice thickness $-3.6 \mathrm{~mm}$.

The entire dataset underwent preliminary processing using the NordicNeuroLab software (NordiclCE, Norway). This included automatic correction of motion artefacts, correction of pre- and post-contrast data in the dynamic series, concentration of contrast agent in brain tissue calculation using relative signal change and T1 mapping. Individual vascular input functions were derived semi-automatically from the superior sagittal sinus [44]). The haematocrit, contrast agent dose and relaxivity of the contrast agent was set individually for each patient. The Patlak pharmacokinetic model was used to assess the low BBB permeability in CSVD resulting in Ktrans (volume transfer coefficient), Vp (fractional blood plasma volume) maps, and AUC (area under the curve - corresponding to increased contrast transit time in the brain) maps.

Once permeability parameter maps were obtained, further data processing was performed in SPM12 (http://www.fil.ion.ucl.ac.uk/spm). This included the following steps: coregistration of each subject's permeability parameter maps and the $\mathrm{T} 1$ images; segmenting the $\mathrm{T} 1$ images into grey matter and white matter, followed by correction of the obtained images using WMH masks based on a MatLab script (https://matlab.ru/), resulting in the binary images of the corrected grey and white matter. Permeability parameters were calculated in ITK-SNAP (http://itksnap.org) separately for the grey matter, NAWM and WMH by superimposing the relevant masks over the individual permeability maps. 
Statistical analysis was performed using IBM SPSS 23.0 (IBM SPSS Statistics, version 23.0, IBM Corp., Armonk, NY, USA) and R 3.4.3 (R Foundation for Statistical Computing, Vienna, Austria) software. Data are presented as $n(\%)$ for categorical variables or as mean \pm standard deviation (SD) or median [interquartile range (IQR)] for quantitative data. Differences between groups were determined using $\chi 2$, independent samples t-test, univariate analysis of variance or Kruskal-Wallis test where appropriate. In all cases, two-way statistical criteria were used. The null hypothesis was rejected if $p<0.05$. Pearson's correlation coefficient and Spearman's correlation were used to assess the relationship between parameters.

\section{Results}

CSVD and the control groups were matched for age and gender, and consisted predominantly of women (Table 1). Vascular risk factors were comparable except for AH which was the dropout criteria for the control group. 
Table 1

Main demographic parameters and risk factors in patients with CSVD and controls

\begin{tabular}{|c|c|c|c|}
\hline Parameters & $\begin{array}{l}\text { CSVD } \\
(n=53)\end{array}$ & $\begin{array}{l}\text { Control group } \\
(n=17)\end{array}$ & $\mathbf{p}$ \\
\hline Gender, women $(n, \%)$ & $37(69.8 \%)$ & $12(70.5 \%)$ & 0.951 \\
\hline Age, years $($ mean $\pm S D)$ & $60.08 \pm 6.8$ & $56.71 \pm 6.7$ & 0.079 \\
\hline $\mathrm{AH}(\mathrm{n}, \%)$ & $45(84.8 \%)$ & & \\
\hline Degree of $\mathrm{AH}(\mathrm{n}, \%)$ & $12(22.6 \%)$ & - & \\
\hline grade 1 & $12(22.6 \%)$ & - & \\
\hline grade 2 & $21(39.6 \%)$ & - & \\
\hline \multicolumn{4}{|l|}{ grade 3} \\
\hline AHT (n, \%) & $4(8.9 \%)$ & - & \\
\hline Irregular use & $11(24.4 \%)$ & - & \\
\hline 1 drug & $20(44.4 \%)$ & - & \\
\hline 2 drugs & $9(20 \%)$ & - & \\
\hline 3 drugs & $1(2.3 \%)$ & - & \\
\hline \multicolumn{4}{|l|}{$\geq 4$ drugs } \\
\hline DM type $2(n, \%)$ & $10(18.9 \%)$ & $0(0 \%)$ & 0.053 \\
\hline $\begin{array}{l}\text { Hypercholesterolemia (total cholesterol } \\
>6,2 \mathrm{mmol} / \mathrm{L} \text { or statin use) }(\mathrm{n}, \%)\end{array}$ & $31(58.5 \%)$ & $8(47 \%)$ & 0.345 \\
\hline Obesity (body mass index $\left.>30 \mathrm{~kg} / \mathrm{m}^{2}\right)(\mathrm{n}, \%)$ & $22(41.5 \%)$ & $5(29.4 \%)$ & 0.373 \\
\hline Smoking (n, \%) & $13(24.5 \%)$ & 7 (41.2\%) & 0.186 \\
\hline
\end{tabular}

[Table 1 near here]

Most of the patients in the main group (84.8\%) had AH of varying severity and were taking one or more antihypertensive drugs.

The main disease symptoms were $\mathrm{Cl}$, gait disturbances unrelated to post-stroke hemiparesis, and MRI changes including WMH, lacunes, microbleeds and dilated perivascular spaces (Table 2). 
Table 2

Clinical symptoms and MRI signs in patients with CSVD.

\begin{tabular}{|c|c|}
\hline Parameters & $\begin{array}{l}\text { CSVD } \\
(n=53)\end{array}$ \\
\hline$C l(\mathrm{n}, \%):$ & $53(100 \%)$ \\
\hline subjective & $22(41.5 \%)$ \\
\hline mild & $24(45.3 \%)$ \\
\hline dementia & $7(13.2 \%)$ \\
\hline Gait disturbances, unrelated to hemiparesis (n, \%): & $29(54.7 \%)$ \\
\hline Urinary disorders (n, \%) & $21(39.6 \%)$ \\
\hline History of stroke $(\mathrm{n}, \%)$ : & $25(37.9 \%)$ \\
\hline WMH, Fazekas Scale (n, \%) & $16(30.2 \%)$ \\
\hline score 1 & $16(30.2 \%)$ \\
\hline score 2 & $21(39.6 \%)$ \\
\hline \multicolumn{2}{|l|}{ score 3} \\
\hline Lacunes (n, \%) & $26(49.1 \%)$ \\
\hline Microbleeds (n, \%) & $25(47.2 \%)$ \\
\hline Perivascular spaces $(\mathrm{n}, \%)$ & $53(100 \%)$ \\
\hline Total CSVD score $(\mathrm{n}, \%)$ & $0(0 \%)$ \\
\hline 1 sign & $19(35.8 \%)$ \\
\hline 2 signs & $25(47.3 \%)$ \\
\hline 3 signs & $9(16.9 \%)$ \\
\hline \multicolumn{2}{|l|}{4 signs } \\
\hline Total WMH, sm ${ }^{3}$ & $13830[5747 ; 32145]$ \\
\hline
\end{tabular}

[Table 2 near here]

Differences in mean asleep DBP, and asleep SBP and DBP load were found when ABPM results were compared between subjects with CSVD and those in the control group (Table 3). 
Table 3

ABPM results in subjects with CSVD and controls.

\begin{tabular}{|llll|}
\hline Parameters & CSVD & Control group & $\mathbf{p}$ \\
& $(\mathbf{n}=\mathbf{5 3})$ & $(\mathbf{n = 1 7 )}$ & \\
\hline 24-h SBP $(\mathrm{mmHg})$ & $120.1[112.9 ; 127.5]$ & $118.3[110.1 ; 121.2]$ & 0.160 \\
\hline 24-h DBP $(\mathrm{mmHg})$ & $78.8[73.3 ; 86.8]$ & $75.9[71.7 ; 76.6]$ & 0.091 \\
\hline Mean awake SBP $(\mathrm{mmHg})$ & $122.8[114.4 ; 132.4]$ & $119.0[111.7 ; 125.3]$ & 0.386 \\
\hline Mean awake DBP $(\mathrm{mmHg})$ & $81.9[76.3 ; 91.3]$ & $77.4[74.2 ; 78.3]$ & 0.903 \\
\hline Maximal awake SBP $(\mathrm{mmHg})$ & $146[137 ; 164]$ & $142[134 ; 148]$ & 0.072 \\
\hline Maximal awake DBP $(\mathrm{mmHg})$ & $105.0[94.0 ; 112.0]$ & $99.0[92.0 ; 106.0]$ & 0.217 \\
\hline Awake SBP load $(\%)$ & $4.6[0.0 ; 20.8]$ & $0.9[0.0 ; 3.9]$ & 0.131 \\
\hline Awake DBP load $(\%)$ & $13.6[1.2 ; 54.6]$ & $6.2[0.8 ; 14.1]$ & 0.075 \\
\hline Awake SD of SBP $(\mathrm{mmHg})$ & $10.1[8.1 ; 13.8]$ & $9.4[7.9 ; 10.9]$ & 0.381 \\
\hline Awake SD of DBP $(\mathrm{mmHg})$ & $9.3[7.2 ; 11.5]$ & $9.7[7.4 ; 11.6]$ & 0.903 \\
\hline Mean asleep SBP $(\mathrm{mmHg})$ & $114.0[106.4 ; 120.9]$ & $110.3[102.7 ; 115.9]$ & 0.169 \\
\hline Mean asleep DBP $(\mathrm{mmHg})$ & $72.9[66.7 ; 80.0]$ & $67.2[64.4 ; 64.4]$ & 0.000 \\
\hline Maximal asleep SBP $(\mathrm{mmHg})$ & $128.0[124.0 ; 141.0]$ & $125.0[119.0 ; 136.0]$ & 0.311 \\
\hline Maximal asleep DBP $(\mathrm{mmHg})$ & $87.0[78.0 ; 96.0]$ & $83.0[76.0 ; 89.0]$ & 0.103 \\
\hline Asleep SBP load $(\%)$ & $17.3[5.4 ; 45.0]$ & $5.8[0.0 ; 18.8]$ & 0.009 \\
\hline Asleep DBP load $(\%)$ & $57.2[18.6 ; 94.4]$ & $17.5[13.8 ; 22.7]$ & 0.002 \\
\hline Asleep SD of SBP $(\mathrm{mmHg})$ & $8.2[5.9 ; 10.0]$ & $8.4[6.9 ; 10.8]$ & 0.732 \\
\hline Asleep SD of DBP $(\mathrm{mmHg})$ & $8.1[6.3 ; 10.0]$ & $8.6[7.2 ; 10.9]$ & 0.304 \\
\hline
\end{tabular}

[Table 3 near here]

Increased 24-hour, awake and asleep SBP and DBP values during both the day and night had relations with WMH load, based on Fazekas Scale score and volume, and total CSVD score (Table 4). 
Table 4

Relationship between ABPM, WMH, and total CSVD score

\begin{tabular}{|c|c|c|c|c|c|c|}
\hline Parameters & $\begin{array}{l}\text { Fazekas } \\
1 \\
(n=16)\end{array}$ & $\begin{array}{l}\text { Fazekas } \\
2 \\
(n=16)\end{array}$ & $\begin{array}{l}\text { Fazekas } \\
3 \\
(n=21)\end{array}$ & $\begin{array}{l}p \\
\text { for Fazekas } \\
\text { score }\end{array}$ & $\begin{array}{l}\text { Correlation } \\
\text { with } \\
\text { WMH } \\
{ }^{*} \mathrm{p}<0.05 \\
{ }^{* *} \mathrm{p}<0.01\end{array}$ & $\begin{array}{l}\text { Correlation } \\
\text { with } \\
\text { total CSVD } \\
\text { score } \\
{ }^{*} \mathrm{p}<0.05 \\
{ }^{*} \mathrm{p}<0.01\end{array}$ \\
\hline 24-h SBP (mmHg) & $\begin{array}{l}116.7 \\
{[112.0 ;} \\
121.2]\end{array}$ & $\begin{array}{l}114.5 \\
{[107.6 ;} \\
120.9]\end{array}$ & $\begin{array}{l}126.6 \\
{[124.2 ;} \\
134.0]\end{array}$ & 0.040 & $0.317 *$ & $0.272^{*}$ \\
\hline 24-h DBP (mmHg) & $\begin{array}{l}77.1 \\
{[73.0 ;} \\
80.5]\end{array}$ & $\begin{array}{l}76.1 \\
{[70.4 ;} \\
81.7]\end{array}$ & $\begin{array}{l}85.5 \\
{[80.0 ;} \\
91.4]\end{array}$ & 0.031 & 0.287 & $0.290 *$ \\
\hline $\begin{array}{l}\text { Mean awake SBP } \\
(\mathrm{mmHg})\end{array}$ & $\begin{array}{l}119.5 \\
{[111.9 ;} \\
126.5]\end{array}$ & $\begin{array}{l}122.2 \\
{[114.4 ;} \\
123.8]\end{array}$ & $\begin{array}{l}129.7 \\
{[123.5 ;} \\
137.3]\end{array}$ & 0.384 & 0.288 & $0.243^{*}$ \\
\hline $\begin{array}{l}\text { Mean awake DBP } \\
(\mathrm{mmHg})\end{array}$ & $\begin{array}{l}77.9 \\
{[75.9} \\
86.6]\end{array}$ & $\begin{array}{l}80.0 \\
{[75.7 ;} \\
84.9]\end{array}$ & $\begin{array}{l}88.8 \\
{[81.3 ;} \\
93.5]\end{array}$ & 0.59 & 0.292 & $0.339 * *$ \\
\hline $\begin{array}{l}\text { Maximal awake } \\
\text { SBP }(\mathrm{mmHg})\end{array}$ & $\begin{array}{l}140 \\
{[135 ;} \\
152]\end{array}$ & $\begin{array}{l}145 \\
{[140 ;} \\
151]\end{array}$ & $\begin{array}{l}161 \\
{[144 ;} \\
170]\end{array}$ & 0.038 & $0.332^{*}$ & $0.274^{*}$ \\
\hline $\begin{array}{l}\text { Maximal awake } \\
\text { DBP }(\mathrm{mmHg})\end{array}$ & $\begin{array}{l}96.5 \\
{[91.0 ;} \\
107.5]\end{array}$ & $\begin{array}{l}102.5 \\
{[93.0 ;} \\
110.0]\end{array}$ & $\begin{array}{l}111.0 \\
{[97.0 ;} \\
118.0]\end{array}$ & 0.053 & $0.323^{*}$ & 0.176 \\
\hline Awake SBP load (\%) & $\begin{array}{l}0.2 \\
{[0.0 ; 6.6]}\end{array}$ & $\begin{array}{l}1.0 \\
{[0.0 ; 7.8]}\end{array}$ & $\begin{array}{l}16.8 \\
{[5.4 ;} \\
45.0]\end{array}$ & 0.110 & 0.185 & $0.284^{*}$ \\
\hline Awake DBP load (\%) & $\begin{array}{l}31.8 \\
{[18.9 ;} \\
64.9]\end{array}$ & $\begin{array}{l}52.4 \\
{[6.2 ;} \\
87.6]\end{array}$ & $\begin{array}{l}94.0 \\
{[47.5 ;} \\
100.0]\end{array}$ & 0.130 & 0.284 & $0.284^{*}$ \\
\hline $\begin{array}{l}\text { Awake SD of SBP } \\
(\mathrm{mmHg})\end{array}$ & $\begin{array}{l}9.7 \\
{[8.0 ;} \\
12.9]\end{array}$ & $\begin{array}{l}9.9 \\
{[7.3 ;} \\
12.2]\end{array}$ & $\begin{array}{l}11.9 \\
{[8.8 ;} \\
13.9]\end{array}$ & 0.369 & -0.003 & 0.129 \\
\hline
\end{tabular}




\begin{tabular}{|c|c|c|c|c|c|c|}
\hline Parameters & $\begin{array}{l}\text { Fazekas } \\
1 \\
(n=16)\end{array}$ & $\begin{array}{l}\text { Fazekas } \\
2 \\
(n=16)\end{array}$ & $\begin{array}{l}\text { Fazekas } \\
3 \\
(n=21)\end{array}$ & $\begin{array}{l}\text { P } \\
\text { for Fazekas } \\
\text { score }\end{array}$ & $\begin{array}{l}\begin{array}{l}\text { Correlation } \\
\text { with }\end{array} \\
\text { WMH } \\
{ }^{*} \mathrm{p}<0.05 \\
{ }^{*} \mathrm{p}<0.01\end{array}$ & $\begin{array}{l}\text { Correlation } \\
\text { with } \\
\text { total CSVD } \\
\text { score } \\
{ }^{*} p<0.05 \\
{ }^{* *} p<0.01\end{array}$ \\
\hline \multirow{2}{*}{$\begin{array}{l}\text { Awake SD of DBP } \\
(\mathrm{mmHg})\end{array}$} & 3.8 & 6.8 & 41.0 & 0.088 & 0.145 & 0.001 \\
\hline & $\begin{array}{l}{[0.5 ;} \\
36.2]\end{array}$ & $\begin{array}{l}{[1.9 ;} \\
33.7]\end{array}$ & $\begin{array}{l}{[8.5 ;} \\
54.7]\end{array}$ & & & \\
\hline \multirow{2}{*}{$\begin{array}{l}\text { Mean asleep SBP } \\
(\mathrm{mmHg})\end{array}$} & 109.0 & 108.9 & 119.3 & 0.000 & $0.357^{*}$ & $0.255^{*}$ \\
\hline & $\begin{array}{l}\text { [104.3; } \\
\text { 115.6] }\end{array}$ & $\begin{array}{l}\text { [98.9; } \\
111.3]\end{array}$ & $\begin{array}{l}\text { [117.0; } \\
\text { 130.7] }\end{array}$ & & & \\
\hline \multirow{2}{*}{$\begin{array}{l}\text { Mean asleep DBP } \\
(\mathrm{mmHg})\end{array}$} & 68.8 & 70.4 & 80.0 & 0.007 & $0.364^{*}$ & $0.370 * \star$ \\
\hline & $\begin{array}{l}\text { [65.0; } \\
\text { 73.0] }\end{array}$ & $\begin{array}{l}\text { [61.6; } \\
79.4]\end{array}$ & $\begin{array}{l}{[72.7 ;} \\
83.4]\end{array}$ & & & \\
\hline \multirow{2}{*}{$\begin{array}{l}\text { Maximal asleep } \\
\text { SBP }(\mathrm{mmHg})\end{array}$} & 125.5 & 124.0 & 136.0 & 0.001 & 0.087 & 0.161 \\
\hline & $\begin{array}{l}\text { [123.0; } \\
128.0]\end{array}$ & $\begin{array}{l}\text { [113.0; } \\
\text { 135.5] }\end{array}$ & $\begin{array}{l}\text { [129.0; } \\
\text { 154.0] }\end{array}$ & & & \\
\hline \multirow{2}{*}{$\begin{array}{l}\text { Maximal asleep } \\
\text { DBP }(\mathrm{mmHg})\end{array}$} & 82.5 & 82.5 & 91.0 & 0.054 & -0.011 & 0.142 \\
\hline & $\begin{array}{l}{[76.0} \\
89.5]\end{array}$ & $\begin{array}{l}{[73.0} \\
96.0]\end{array}$ & $\begin{array}{l}{[87.0 ;} \\
99.0]\end{array}$ & & & \\
\hline \multirow[t]{2}{*}{ Asleep SBP load (\%) } & 12.7 & 5.5 & 45.0 & 0.000 & $0.441 * *$ & $0.387 * \star$ \\
\hline & $\begin{array}{l}{[5.6} \\
18.9]\end{array}$ & $\begin{array}{l}{[0.0} \\
16.2]\end{array}$ & $\begin{array}{l}\text { [31.3; } \\
98.3]\end{array}$ & & & \\
\hline \multirow[t]{2}{*}{ Asleep DBP load (\%) } & 31.8 & 52.4 & 94.0 & 0.019 & $0.338 *$ & 0.391 ** \\
\hline & $\begin{array}{l}{[18.9} \\
64.9]\end{array}$ & $\begin{array}{l}{[6.2 ;} \\
87.6]\end{array}$ & $\begin{array}{l}{[47.5 ;} \\
100.0]\end{array}$ & & & \\
\hline \multirow{2}{*}{$\begin{array}{l}\text { Asleep SD of SBP } \\
(\mathrm{mmHg})\end{array}$} & 6.9 & 8.3 & 8.7 & 0.791 & 0.087 & -0.144 \\
\hline & $\begin{array}{l}{[5.1 ;} \\
10.6]\end{array}$ & {$[7.3 ; 9.3]$} & $\begin{array}{l}{[5.9 ;} \\
12.9]\end{array}$ & & & \\
\hline \multirow{2}{*}{$\begin{array}{l}\text { Asleep SD of DBP } \\
(\mathrm{mmHg})\end{array}$} & 8.2 & 8.6 & 7.3 & 0.852 & -0.011 & -0.202 \\
\hline & $\begin{array}{l}\text { [5.9; } \\
11.0]\end{array}$ & {$[6.4 ; 9.7]$} & {$[6.8 ; 9.6]$} & & & \\
\hline
\end{tabular}

[Table 4 near here] 
To clarify the link between daily BP fluctuations and BBB damage, BBB permeability was assessed using DCE-MRI in patients and controls (Table 5). CSVD was characterized by increased BBB permeability. The Ktrans in GM, Vp and AUC in GM and NAWM differed significantly between patients with CSVD and the controls.

Table 5

DCE-MRI parameters in patients with CSVD and controls.

\begin{tabular}{|llll|}
\hline Parameters & $\begin{array}{l}\text { CSVD } \\
(\mathbf{n}=\mathbf{5 3})\end{array}$ & $\begin{array}{l}\text { Control group } \\
(\mathbf{n}=\mathbf{1 7})\end{array}$ & $\mathbf{p}$ \\
\hline Ktrans GM $\left(\mathrm{min}^{-1}\right)$ & $0.0002[0.0001 ; 0.0004]$ & $0.0002[0.0001 ; 0.0002]$ & $\mathbf{0 . 0 4 2}$ \\
\hline Vp, GM & $1.2836[1.0199 ; 1.7332]$ & $1.0126[0.7797 ; 1.3264]$ & $\mathbf{0 . 0 1 3}$ \\
\hline AUC, GM & $0.0031[0.0025 ; 0.0041]$ & $0.0022[0.0019 ; 0.0026]$ & $\mathbf{0 . 0 0 0}$ \\
\hline Ktrans, NAWM $\left(\mathrm{min}^{-1}\right)$ & $0.0001[0.0000 ; 0.0001]$ & $0.0001[0.0000 ; 0.0001]$ & 0.735 \\
\hline Vp, NAWM & $0.5609[0.4076 ; 0.7039]$ & $0.4265[0.3457 ; 0.4573]$ & $\mathbf{0 . 0 2 3}$ \\
\hline AUC, NAWM & $0.0013[0.0011 ; 0.0015]$ & $0.0011[0.0009 ; 0.0012]$ & $\mathbf{0 . 0 0 2}$ \\
\hline Ktrans, WMH $\left(\mathrm{min}^{-1}\right)$ & $0.0001[0.0000 ; 0.0002]$ & - & - \\
\hline Vp, WMH & $0.4847[0.3320 ; 0.8198]$ & - & - \\
\hline AUC, WMH & $0.0012[0.0009 ; 0.0019]$ & - & - \\
\hline
\end{tabular}

[Table 5 near here]

BBB permeability decreased as WMH Fazekas score increased, with significant differences in Vp and AUC in WMH (Table 6). 
Table 6

DCE-MRI parameters based on the Fazekas stage

\begin{tabular}{|c|c|c|c|c|}
\hline Parameters & $\begin{array}{l}\text { Fazekas } 1 \\
(n=16)\end{array}$ & $\begin{array}{l}\text { Fazekas } 2 \\
(n=16)\end{array}$ & $\begin{array}{l}\text { Fazekas } 3 \\
(n=21)\end{array}$ & p \\
\hline Ktrans GM $\left(\min ^{-1}\right)$ & $\begin{array}{l}0.0003[0.0002 ; \\
0.0004]\end{array}$ & $\begin{array}{l}0.0003 \\
{[0.0001 ; 0.0005]}\end{array}$ & $\begin{array}{l}0.0002 \\
{[0.0001 ; 0.0004]}\end{array}$ & 0.946 \\
\hline Vp GM & $1.6954[1.0997 ; 2.0808]$ & $\begin{array}{l}1.2464 \\
{[1.0814 ; 1.6571]}\end{array}$ & $\begin{array}{l}1.1579 \\
{[1.0045 ; 1.5523]}\end{array}$ & 0.182 \\
\hline AUC GM & $0.0035[0.0025 ; 0.0044]$ & $\begin{array}{l}0.0033 \\
{[0.0028 ; 0.0039]}\end{array}$ & $\begin{array}{l}0.0031 \\
{[0.0026 ; 0.0033]}\end{array}$ & 0.570 \\
\hline $\begin{array}{l}\text { Ktrans NAWM (min } \\
-1 \text { ) }\end{array}$ & $0.0001[0.0000 ; 0.0001]$ & $\begin{array}{l}0.0000 \\
{[0.0000 ; 0.0001]}\end{array}$ & $\begin{array}{l}0.0001 \\
{[0.0000 ; 0.0001]}\end{array}$ & 0.361 \\
\hline Vp NAWM & $0.7074[0.4245 ; 0.7543]$ & $\begin{array}{l}0.5570 \\
{[0.4169 ; 0.5999]}\end{array}$ & $\begin{array}{l}0.5212 \\
{[0.3893 ; 06281]}\end{array}$ & 0.420 \\
\hline AUC NAWM & $0.0013[0.0011 ; 0.0017]$ & $\begin{array}{l}0.0014 \\
{[0.0012 ; 0.0015]}\end{array}$ & $\begin{array}{l}0.0012 \\
{[0.0011 ; 0.0015]}\end{array}$ & 0.940 \\
\hline $\begin{array}{l}\text { Ktrans WMH ( } \text { min }^{-} \\
\text {1) }\end{array}$ & $0.0001[0.0001 ; 0.0003]$ & $\begin{array}{l}0.0001 \\
{[0.0000 ; 0.0002]}\end{array}$ & $\begin{array}{l}0.0001 \\
{[0.0000 ; 0.0001]}\end{array}$ & 0.563 \\
\hline Vp WMH & $0.9323[0.4574 ; 1.5027]$ & $\begin{array}{l}0.5266 \\
{[0.4054 ; 0.6131]}\end{array}$ & $\begin{array}{l}0.4041 \\
{[0.2823 ; 0.5034]}\end{array}$ & 0.003 \\
\hline AUC WMH & $0.0021[0.0012 ; 0.0025]$ & $\begin{array}{l}0.0013 \\
{[0.0010 ; 0.0017]}\end{array}$ & $\begin{array}{l}0.0010 \\
{[0.0008 ; 0.0012]}\end{array}$ & 0.001 \\
\hline
\end{tabular}

[Table 6 near here]

Statistically significant correlations were found between AUC in GM and NAWM and Vp in NAWM and parameters of 24-h and awake SBP and DBP. AUC in NAWM also correlated with parameters of asleep SBP and DBP (Table 7). 
Table 7

Correlation between ABPM results and DCE-MRI results $\left({ }^{*} p<0.05\right.$, $\star * \mathrm{p}<0.01)$

\begin{tabular}{|llll|}
\hline Parameters & AUC, GM & Vp, NAWM & AUC, NAWM \\
\hline 24-h SBP & 0.148 & $\mathbf{0 . 2 5 4 *}$ & $\mathbf{0 . 3 2 1 * *}$ \\
\hline 24-h DBP & 0.201 & 0.226 & $\mathbf{0 . 2 8 3 *}$ \\
\hline Mean awake DBP & $\mathbf{0 . 2 5 1 *}$ & $\mathbf{0 . 2 4 5 *}$ & $\mathbf{0 . 3 3 1 * *}$ \\
\hline Maximal awake SBP & $\mathbf{0 . 2 3 9 *}$ & $\mathbf{0 . 3 3 1 * *}$ & $\mathbf{0 . 3 2 5 * *}$ \\
\hline Maximal awake DBP & $\mathbf{0 . 2 6 5 *}$ & $\mathbf{0 . 3 4 4 * *}$ & $\mathbf{0 . 3 3 6 * *}$ \\
\hline Awake SBP load (\%) & $\mathbf{0 . 2 8 4 *}$ & $\mathbf{0 . 3 4 5 * *}$ & $\mathbf{0 . 4 5 9 * *}$ \\
\hline Mean asleep SBP & 0.081 & 0.174 & $\mathbf{0 . 2 3 7 *}$ \\
\hline Maximal asleep SBP & 0.051 & 0.173 & $\mathbf{0 . 2 3 7 *}$ \\
\hline Maximal asleep DBP & 0.142 & 0.188 & $\mathbf{0 . 2 5 6 *}$ \\
\hline
\end{tabular}

[Table 7 near here]

No significant correlations were found between ABPM results and BBB permeability parameters in WMH, so these data are not provided.

\section{Discussion}

This study sought to clarify the relation between the 24-hour BP profile and DCE-MRI BBB permeability, in patients with CSVD, most of whom with a long-standing $A H$. ABPM results showed a good response to AHT in the main group. Most of ABPM parameters in the study (except for mean asleep DBP, asleep SBP load (\%), and asleep DBP load (\%), did not show intergroup differences corresponding to doctors' and patients' opinion that AH was well controlled as measured on the outpatient basis. However, comparison of ABPM results with the severity of WMH based on Fazekas Scale score and its volume, as well as with total CSVD score showed direct and significant relations and correlations, respectively. These data indicate the presence of certain mechanisms related to the abnormal ABPM parameters in patients compared with controls. Since a significant proportion of the studied patients had mild AH, as well as mild clinical and MRI signs of the disease, we could assume that BBB damage and its high permeability played a significant role in CSVD development. This hypothesis is based on the study results, that indicate the special role of endothelial dysfunction with high BBB permeability as a mechanism of early CSVD [32; 45; 46; 47]. 
According to DCE-MRI, all the study parameters of BBB permeability in NAWM and GM, except for Ktrans in NAWM, were significantly higher in patients with CSVD than in controls. This data confirms the results of previous studies using DCE-MRI in patients with CSVD, which showed increased BBB permeability in the cortical and deep GM, NAWM adjacent to the WMH, and in the WMH themselves, with no correlation with the severity of the latter $[32 ; 35 ; 48]$. At the same time, Wardlaw et al showed a link between increased BBB permeability and the severity of white matter abnormalities, $\mathrm{AH}$ and elevated pulse pressure [32]. The peculiar features of patients with CSVD and AH were absence of significant differences between the parameters of BBB permeability in GM and NAWM depending on WMH Fazekas Score. These results may be connected with the well-preserved small vessel endothelium in NAWM and GM in CSVD and agree with the previously obtained data about the universal mechanism of BBB damage in CSVD in patients with AH of varying severity [30]. On the other hand, the obtained data about the significant reduction in BBB permeability with increasing WMH may correspond to the conditions that characterize late-stage CSVD such as progressive endothelial death, impaired autoregulation due to small vessel remodelling, wall thickening and lumen narrowing, reduced microvasculatory perfusion [15; 35]. This explanation is also supported by the fact that none of the abnormal ABPM parameters had relations with increased BBB permeability in the WMH. AUC, which characterizes the contrast delay in the brain, had the highest sensitivity out of BBB permeability parameters. This result matches the different responses to elevated BP in normotensive and hypertensive rats in experiment: the hypertensive rats had higher permeability to sucrose which was absorbed more slowly by the brain, and the authors attributed this to changes in blood flow in AH [26; 49]. Increased BBB permeability, as assessed by AUC, was connected with mean awake DBP, maximal awake SBP and DBP, awake SBP load (\%) in GM, as well as with mean awake DBP and asleep SBP, maximal awake and asleep SBP and DBP, awake SBP load (\%) in the NAWM. Although ABPM parameters, which had correlations with increased BBB permeability in the main group, did not differ from the control group, we cannot exclude preceding rises in BP that exceeds the upper threshold of cerebral autoregulation, which is associated with BBB damage and its high permeability [29]. It can be assumed that the use of AHT may change the upper threshold of cerebral autoregulation and the conditions for its disruption, which is indirectly supported by the fact that a greater reduction in BP in elderly people with $\mathrm{AH}$ is associated with increased cerebral blood flow (CBF), corresponding to a shift in the autoregulation curve [50]. On the whole, these data support the necessity for more aggressive treatment of AH in patients with CSVD [51; 52]. The risk of cardiovascular complications is decreased when BP is reduced more aggressively, so the guidelines were rationally revised to the target $\mathrm{SBP}<120 \mathrm{mmHg}$ [53]. A recent randomized trial also supported this finding, as cerebral perfusion did not decrease in patients with severe CSVD when BP was aggressively reduced, unlike in healthy controls [54].

The obtained data about the universal nature of increased BBB permeability in the NAWM and GM in patients with CSVD indicate an ongoing pathological process in the small arteries, which leads to endothelial damage in relatively well-preserved small vessels. The connection between BBB permeability in NAWM and GM and elevated ABPM parameters indicated the importance of autoregulation dysfunction in promoting this mechanism. It is possible that the underestimation of the pathological 
mechanism of brain damage due to increased BBB permeability can partly explain the significance of $\mathrm{AH}$ in middle age for the development of $\mathrm{Cl}$ in the elderly $[55 ; 56]$.

The lack of significant differences in ABPM results related to BBB permeability between patients and controls allow us to hypothesize the presence of additional factors of endothelial damage and increased BBB permeability alongside AH. These factors may be chronic inflammation [57] or high salt sensitivity, which have been found to independently correlate with CSVD [58].

The obtained data indicate the necessity for more aggressive treatment of $\mathrm{AH}$ and repeat usage of $\mathrm{ABPM}$ as well as requirement for searching and affecting factors, that potentiate the role of AH in CSVD development. It is obvious that further studies are needed on the effect of AHT on BBB permeability and its ability to protect the brain from damage in patients with CSVD.

\section{Abbreviations}

CSVD - cerebral small vessel disease

$\mathrm{Cl}$ - cognitive impairment

BBB - blood-brain barrier

$\mathrm{AH}$ - arterial hypertension

AHT - antihypertensive therapy

ABPM - ambulatory blood pressure monitoring

BP - blood pressure

SBP - systolic blood pressure

DBP - diastolic bloop pressure

DM - diabetes mellitus

$\mathrm{WMH}$ - white matter hyperintensity

NAWM - normal-appearing white matter

GM - grey matter

MRI - magnetic resonance imaging

DCE-MRI - T1-weighted dynamic contrast-enhanced magnetic resonance imaging Ktrans - volume transfer coefficient 
Vp - fractional blood plasma volume

AUC - area under the curve

\section{Declarations}

\section{Acknowledgements.}

MRI examinations was performed using the scanner at the Structural and Functional Brain Mapping Centre for Collective Use at the Research Centre of Neurology.

\section{Data availability statement}

Raw data were generated at Research Center of Neurology. The data that support the findings of this study are available from the corresponding author upon reasonable request. Clinical, neurovisualization and statistical data will be available upon request from any qualified investigator.

\section{Authors' contributions}

LAD, KVS, EIK, MRZ, BMA, EVG, MVK had full access to all the data in the study and takes responsibility for the integrity of the data and the accuracy of the data analysis. Study concept and design - LAD. Acquisition of data - all authors. Data analysis and interpretation of data - LAD, EIK, KVS, MRZ and BMA. Drafting of the manuscript - LAD, KVS, EIK. Critical revision of the manuscript for important intellectual content - all authors. Statistical analysis - KVS, MRZ and BMA. Study supervision: LAD EVG and MVK.

\section{Competing interests}

The authors declare that they have no competing interests.

\section{References}

1. Gorelick, P.B. et al. Vascular contributions to cognitive impairment and dementia: a statement for healthcare professionals from the American Heart Association / American Stroke Association. Stroke. 42 (9), 2672-2713; 10.1161/STR.0b013e3182299496 (2011)

2. Deramecourt, V. et al. Staging and natural history of cerebrovascular pathology in dementia. Neurology. 78, 1043-1050; 10.1212/WNL.0b013e31824e8e7f (2012)

3. Azarpazhooh, M.R. et al. Concomitant vascular and neurodegene0rative pathologies double the risk of dementia. Alzheimers Demen. 14 (2), 148-156; 10.1016/j.jalz.2017.07.755 (2018)

4. Dufouil, C. et al. Longitudinal study of blood pressure and white matter hyperintensities: the EVA MRI cohort. Neurology. 56, 921-926; 10.1212/wnl.56.7.921 (2001)

5. de Leeuw, F.E. et al. Hypertension and cerebral white matter lesions in a prospective cohort study. Brain. 125, 765-772; 10.1093/brain/awf077 (2002) 
6. Wardlaw, J.M., Smith, C., Dichgans, M. Mechanisms underlying sporadic cerebral small vessel disease: Insights from neuroimaging. Lancet Neurol. 12, 483-497; 10.1016/S1474-4422(13)70060-7 (2013)

7. Fisher, C.M. Lacunar infarcts - a review. Cerebrovasc Dis. 1, 311-20; https://doi.org/10.1159/000108861 (1991)

8. Lammie, G. A., Brannan, F., Slattery, J., Warlow, C.. Nonhypertensive cerebral small-vessel disease: an autopsy study. Stroke. 28 (11), 2222-2229; 10.1161/01.str.28.11.2222 (1997)

9. Kemper, T.L. et al. Neuropathology of progressive cognitive decline in chronically hypertensive rhesus monkeys. Acta Neuropathol. 101 (2), 145-53; 10.1007/s004010000278 (2001)

10. Bailey, E.L., McCulloch, J., Sudlow, C., Wardlaw, J.M. Potential animal models of lacunar stroke. A systematic review. Stroke. 40 (6), 451-8; 10.1161/STROKEAHA.108.528430 (2009)

11. van Dijk, E.J. et al. The association between blood pressure, hypertension, and cerebral white matter lesions: cardiovascular determinants of dementia study. Hypertension. 44 (5), 625-30; 10.1161/01.HYP.0000145857.98904.20 (2004)

12. Debette, S., Markus, H.S. The clinical importance of white matter hyperintensities on brain magnetic resonance imaging: systematic review and meta-analysis. BMJ. 341, c3666; 10.1136/bmj.c3666 (2010)

13. Poggesi, A. et al. 2001-2011: a decade of the LADIS (Leukoaraiosis And DISability) Study: what have we learned about white matter changes and small-vessel disease? Cerebrovasc dis. 32 (6), 577-588; 10.1159/000334498 (2011)

14. Fisher, C.M. The arterial lesions underlying lacunes. Acta Neuropathol. 12 (1), 1-15; 10.1007/BF00685305 (1969)

15. McAleese, K.E. et al. Post-mortem assessment in vascular dementia: advances and aspirations. BMC med. 14 (1), 129. 10.1186/s12916-016-0676-5 (2016)

16. $\mathrm{Ma}$, Y. et al. Blood pressure variability and cerebral small vessel disease: a systematic review and meta-analysis of population-based cohorts. Stroke. 51 (1), 82-89;

10.1161/STROKEAHA.119.026739 (2020)

17. Tully, P.J. et al. Association Between Blood Pressure Variability and Cerebral Small-Vessel Disease: A Systematic Review and Meta-Analysis. J Am Heart Assoc. 9 (1), e013841;

10.1161/JAHA.119.013841 (2020)

18. Arima, H., Chalmers, J. PROGRESS: prevention of recurrent stroke. J Clin Hypertens. 13 (9), 693-702; 10.1111/j.1751-7176.2011.00530.x (2011)

19. Hankey, G. J. The global and regional burden of stroke. Lancet Glob Health. 1 (5), e239-e240

; 10.1016/S2214-109X(13)70095-0 (2013)

20. Weber, R. et al. Telmisartan on top of antihypertensive treatment does not prevent progression of cerebral white matter lesions in the prevention regimen for effectively avoiding second strokes (PRoFESS) MRI substudy. Stroke. 43 (9), 2336-2342; 10.1161/STROKEAHA.111.648576 (2012) 
21. Williamson, J.D. et al. Effect of intensive vs standard blood pressure control on probable dementia: a randomized clinical trial. JAMA. 321 (6), 553-561; 10.1001/jama.2018.21442 (2019)

22. Mamo, J.C.L. et al. Antihypertensive agents do not prevent blood-brain barrier dysfunction and cognitive deficits in dietary-induced obese mice. Int. J. Obes. 41 (6), 926-934; 10.1038/ijo.2017.57 (2017)

23. Fan, Y. et al. Tight junction disruption of blood-brain barrier in white matter lesions in chronic hypertensive rats. Neuroreport. 26 (17), 1039-43; 10.1097/WNR.0000000000000464 (2015)

24. Wardlaw, J.M., Valdés Hernández, M.C., Muñoz-Maniega, S. What are white matter hyperintensities made of? Relevance to vascular cognitive impairment. J Am Heart Assoc. 4 (6), 001140; 10.1161/JAHA.114.001140 (2016)

25. Iadecola, C. et al. Impact of Hypertension on Cognitive Function: A Scientific Statement From the American Heart Association. Hypertension. 68 (6), e67-e94; 10.1161/HYP.0000000000000053 (2016)

26. Katsi, V. et al. Blood-brain barrier dysfunction: the undervalued frontier of hypertension. J Hum Hypertens. 34 (10), 682-91; https://doi.org/10.1038/s41371-020-0352-2 (2020)

27. Biancardi, V.C., Son, S.J., Ahmadi S., Filosa, J.A., Stern, J.E. Circulating angiotensin II gains access to the hypothalamus and brain stem during hypertension via breakdown of the blood-brain barrier. Hypertension. 63 (3), 572-9; 10.1161/HYPERTENSIONAHA.113.01743 (2014)

28. Hazama, A.F., Amano, S., Ozaki, T. Pathological changes of cerebral vessel endothelial cells in spontaneously hypertensive rats, with special reference to the role of these cells in the development of hypertensive cerebrovascular lesions. Adv Neurol, 20, 359-369 (1978)

29. Iadecola, C., Davisson, R.L. Hypertension and cerebrovascular dysfunction. Cell metab, 7 (6), $476-$ 484; 10.1016/j.cmet.2008.03.010 (2008)

30. Schreiber, S., Bueche, C.Z., Garz, C., Braun, H. Blood brain barrier breakdown as the starting point of cerebral small vessel disease? - New insights from a rat model. Exp Transl Stroke Med. 5 (1), 4; 10.1186/2040-7378-5-4 (2013)

31. Tomimoto, $\mathrm{H}$. et al. Alterations of the blood-brain barrier and glial cells in white-matter lesions in cerebrovascular and Alzheimer's disease patients. Stroke. 27 (11), 2069-2074;

10.1161/01.str.27.11.2069 (1996)

32. Wardlaw, J.M. et al. Blood-brain barrier failure as a core mechanism in cerebral small vessel disease and dementia: evidence from a cohort study. Alzheimers Dement. 13 (6), 634-643;

10.1016/j.jalz.2016.09.006 (2017)

33. Thrippleton, M.J. et al. Quantifying blood-brain barrier leakage in small vessel disease: Review and consensus recommendations. Alzheimers Dement. 15 (6), 840-858; 10.1016/j.jalz.2019.01.013 (2019)

34. Topakian, R., Barrick, T.R., Howe, F.A., Markus, H.S. Blood-brain barrier permeability is increased in normal-appearing white matter in patients with lacunar stroke and leucoaraiosis. J Neurol Neurosurg Psychiatry. 81 (2), 192-197; 10.1136/jnnp.2009.172072 (2010) 
35. Huisa, B.N. et al. Long-Term Blood-Brain Barrier Permeability Changes in Binswanger Disease. Stroke. 46 (9), 2413-2418; 10.1161/STROKEAHA.115.009589 (2015)

36. Wardlaw, J.M. et al. Neuroimaging standards for research into small vessel disease and its contribution to ageing and neurodegeneration. The Lancet Neurol. 12 (8), 822-38; 10.1016/S14744422(13)70124-8 (2013)

37. Albert, M.S. et al. The diagnosis of mild cognitive impairment due to Alzheimer's disease: Recommendations from the National Institute on Aging-Alzheimer's Association workgroups on diagnostic guidelines for Alzheimer's disease. Alzheimers Dement. 7 (3), 270-9; 10.1016/j.jalz.2011.03.008 (2011)

38. McKhann, G.M. et al. The diagnosis of dementia due to Alzheimer's disease: Recommendations from the National Institute on Aging-Alzheimer's Association workgroups on diagnostic guidelines for Alzheimer's disease. Alzheimers Dement. 7 (3), 263-9; 10.1016/j.jalz.2011.03.00 (2011)

39. O’Brien, E. et al. European Society of Hypertension position paper on ambulatory blood pressure monitoring. Journal of hypertension, 31 (9), 1731-1768; 10.1097/HJH.0b013e328363e964 (2013)

40. Mancia, G. et al. 2013 ESH/ESC guidelines for the management of arterial hypertension: the Task Force for the Management of Arterial Hypertension of the European Society of Hypertension (ESH) and of the European Society of Cardiology (ESC). J Hypertens. 31 (7), 1281-357;

10.1097/01.hjh.0000431740.32696.cc (2013)

41. Klarenbeek, P., van Oostenbrugge, R.J., Rouhl, R.P., Knottnerus, I.L., Staals, J. Ambulatory blood pressure in patients with lacunar stroke: association with total MRI burden of cerebral small vessel disease. Stroke. 44 (11), 2995-2999; 10.1161/STROKEAHA.113.002545 (2013)

42. Staals, J. et al. Total MRI load of cerebral small vessel disease and cognitive ability in older people. Neurobiol Aging. 36 (10), 2806-2811; 10.1016/j.neurobiolaging.2015.06.024 (2015)

43. Fazekas, F. et al. MR signal abnormalities at 1.5 T in Alzheimer's dementia and normal aging. AJR Am J Roentgenol. 8 (3), 421-426; 10.2214/ajr.149.2.351 (1987)

44. Li, Y. et al. Hemodynamic assessments of venous pulsatile tinnitus using 4D-flow MRI. Neurology. 91 (6), e586-e593; 10.1212/WNL.0000000000005948 (2018)

45. Wardlaw, J.M., Sandercock, P.A., Dennis, M.S., Starr, J. Is breakdown of the blood-brain barrier responsible for lacunar stroke, leukoaraiosis, and dementia? Stroke. 34 (3), 806 - 12; 1161/01.STR.0000058480.77236.B3 (2003)

46. Rosenberg, G.A. Neurological diseases in relation to the blood-brain barrier. J Cereb Blood Flow Metab. 32 (7), 1139-51; 10.1038/jcbfm.2011.197 (2012)

47. Rajani, R.M. et al. Reversal of endothelial dysfunction reduces white matter vulnerability in cerebral small vessel disease in rats. Sci. Transl. Med. 10 (448), eaam9507; 10.1126/scitranslmed.aam9507 (2018)

48. Li, Y. et al. Higher blood-brain barrier permeability is associated with higher white matter hyperintensities burden. J Neurol. 264 (7), 1474-1481; 10.1007/s00415-017-8550-8 (2017) 
49. Setiadi, A., May, C.N., Yao, S.T. Ablation of astrocytes in the paraventricular nucleus disrupts the blood-brain barrier and increases blood pressure in rats. FASEB J. 31 (S1), 1010.5; https://doi.org/10.1096/fasebj.31.1_supplement.1010.5 (2017)

50. Tryambake, D. et al. Intensive blood pressure lowering increases cerebral blood flow in older subjects with hypertension. Hypertension. 61 (6), 1309-1315; 10.1161/HYPERTENSIONAHA.112.200972 (2013)

51. SPRINT Research Group et al. A randomized trial of intensive versus standard blood-pressure control. N Engl J Med. 373 (22), 2103-2116; 10.1056/NEJMoa1511939 (2015)

52. Mancia, G. et al. Cardiovascular outcomes at different on-treatment blood pressures in the hypertensive patients of the VALUE trial. Eur Heart J. 37 (12), 955-964; 10.1093/eurheartj/ehv633 (2016).

53. Whelton, P.K. et al. 2017 ACC/AHA/AAPA/ABC/ACPM/AGS/APHA/ASH/ASPC/NMA/PCNA guideline for the prevention, Detection, evaluation, and management of high blood pressure in adults: a Report of the American College of Cardiology/American heart Association. Task force on clinical practice guidelines. Hypertension. <bi>71</bi> (6), 1269-1324; 10.1161/HYP.0000000000000066 (2018)

54. Croall, I. D. et al. Effect of standard vs intensive blood pressure control on cerebral blood flow in small vessel disease: the preserve randomized clinical trial. JAMA neurol. 75 (6), 720-727; 10.1001/jamaneurol.2017.5153 (2018)

55. Whitmer, R.A., Sidney, S., Selby, J., Johnston, S.C., Yaffe, K. Midlife cardiovascular risk factors and risk of dementia in late life. Neurology. 64 (2), 277-281; 10.1212/01.WNL.0000149519.47454.F2 (2005)

56. Walker, K.A. et al. Association of midlife to late-life blood pressure patterns with incident dementia. JAMA. 322 (6), 535-545; 10.1001/jama.2019.10575 (2019)

57. Ihara, M., Yamamoto, Y. Emerging Evidence for Pathogenesis of Sporadic Cerebral Small Vessel Disease. Stroke. 47 (2), 554-60; 10.1161/STROKEAHA.115.009627 (2016)

58. Dobrynina, L.A. et al. The Predictive Value of Salt Sensitivity and Osmotic Fragility in the Development of Cerebral Small Vessel Disease. Int J Mol Sci. 21 (6), 2036; 10.3390/ijms21062036 (2020) 